PREHOSPITAL CARE

\title{
Setting the scene for the paramedic in primary care: a review of the literature
}

L Ball

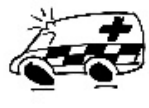

Emerg Med J 2005;22:896-900. doi: 10.1136/emj.2004.019588

Recognition of the paramedic "profession" began in 2003 , with the introduction of statutory registration and the promotion of graduate entry. This paper explores the published evidence which surrounds paramedic practice in an attempt to identify the skills, training, and professional capacity which paramedics of the future will require. A systematic analysis was carried out of key reviews and commentaries published between January 1995 and April 2004, and informal discussions with experts and researchers in the field were undertaken. There remains little high quality published evidence with which to validate many aspects of current paramedic practice. To keep pace with service developments, paramedic training must embrace the complexities of autonomous practice. Undoubtedly in the short term, paramedics must be taught to appropriately identify and manage a far wider range of commonly occurring conditions, minor illnesses, and trauma. However, in the longer term, and more importantly, paramedics must learn to work together to take ownership of the basic philosophies of their practice, which must have their foundation in valid and reliable research.

Correspondence to:

L Ball, Senior Lecturer in Health and Social Care

Research, Sheffield Hallam University, Faculty of Health \& Wellbeing, Room A213 Collegiate Hall, Collegiate Crescent, Sheffield, South Yorkshire S10 2BP, UK; I.ball@shu. ac.uk; BallLinny@aol.com

Accepted for publication 5 November 2004
I n recent times we have seen ambulance services in the UK move away from being a component of secondary care towards the establishment of much firmer links within the primary care setting. Within this framework, the role of the paramedic has also evolved, moving away from its focus on basic first aid and patient transportation, to encompass higher levels of patient care and transform the prognoses of patients suffering from a wide range of trauma and medical conditions.

If ambulance services in UK are to continue to develop and build upon their contribution to the modern National Health Service (NHS), it has been widely acknowledged that they cannot meet this challenge by simply providing more of the same services they provide at present. ${ }^{1}$ The purpose of this review therefore was to examine the current evidence base in emergency medicine, both to inform the content of a third year degree option for the current Paramedic Diploma at Sheffield Hallam University, and to guide the development of a contemporary research agenda for paramedic practice.

\section{DEFINING THE POLICY CONTEXT}

The development of UK emergency services has today moved away from the evaluation of "unnecessary" accident and emergency (A\&E) attendances towards the planning and provision of primary care services which are more responsive to consumer needs. ${ }^{2}$ Consequently, the Department of Health $^{3}$ has summarised the future focus for the development of ambulance services as follows:

- To raise public awareness of what constitutes appropriate use of emergency services.

- To develop and evaluate alternative "emergency" call handling services.

- To review current 999 call prioritisation categories and the management of 999 calls within the Service.

- To create and evaluate alternatives to the routine transport of patients to hospital A\&E departments.

Service initiatives in response to policy Several service initiatives aimed at meeting the challenges listed above are currently under review and evaluation across the UK.

The changing face of paramedic practice In 2000, the Ambulance Service Association ${ }^{4}$ sanctioned the development of "a new echelon of generic health care worker", in the form of the Practitioner in Emergency Care. The impetus towards a broader education for paramedics began in $1998^{3}$ with the adoption of graduate entry to the profession via university undergraduate programmes and moves to establish service links with local universities. Following a national service evaluation by the Commission for Health Improvement, the NHS Modernisation Agency Improvement Partnership for Ambulance Services was launched in September 2003. Its aim was to integrate the ambulance services into the total healthcare system, by developing understanding, building recognition, and releasing staff potential. Having highlighted the need to introduce new ways of working in conjunction with major redesign of the paramedic role, a series of new "practitioner" posts were conceived. These included:

- Community paramedics (based in general practice (GP) surgeries)

- Critical care paramedics (based in hospital intensive care facilities) 
Box 1: Location of UK emergency care practitioner trials

Wave one

- Greater Manchester

- London

- Coventry, Warwickshire, Hereford, and Worcester

- Norfolk, Suffolk, and Cambridgeshire

- County Durham and Darlington

- Devon and Cornwall

Wave two

- Hampshire

- Bedfordshire and Hertfordshire

- Kent, Surrey, and Sussex

- East and North Teesside

- South Yorkshire

- Mersey Region

- Intermediate care paramedics (managing all aspects of the service)

- A\&E paramedics

- Treat and refer paramedics (based in minor injury units/ walk-in centres).

The government's Changing Workforce Programme subsequently launched the paramedic Accelerated Development Programme and the paramedic Practitioner Trial, both of which focused upon the evaluation of a wide range of "emergency care practitioners" in several pilot sites across the UK (box 1).

\section{Telephone helplines}

NHS Direct (1998) and Care Direct (2001) services have been introduced as an alternative to the GP or "999" call, and have been shown to provide high levels of customer satisfaction, although access appears to be limited to certain more affluent and articulate groups within the overall population. ${ }^{5}$ Indeed, their critics have voiced concerns that such facilities have simply created an additional demand for emergency services, rather than rationalising the pre-existing workload. ${ }^{6}$

\section{Minor injury units and walk-in centres}

To provide relief for overcrowded hospital A\&E departments, minor injury units, walk-in centres, and primary care centres have been gradually phased in across the UK since the mid1990s. Studies continue to demonstrate the effectiveness of such services in terms of reduced waiting times and subsequent A\&E attendances, ${ }^{78}$ although to date, their cost effectiveness has not been rigorously assessed. ${ }^{9}$ There remains, however, a wide variation in service provision, which may have given rise to some level of confusion about service availability, in particular among certain minority populations, specially where English is not the first language. ${ }^{9}$

\section{Intermediate care services}

The need to put in place some form of "intermediate" care for older people and those with mental illness gained momentum following a report from the Audit Commission ${ }^{10}$ and the findings of the National Bed Enquiry. ${ }^{11}$ Guidance by the Department of Health ${ }^{12}$ placed emphasis on the development of a "seamless continuum of services", providing rapid assessment and diagnosis, the avoidance of unnecessary hospital admissions and the facilitation of earlier, supported discharge in the home environment. Although there is insufficient evidence to draw definitive conclusions, it does appear that the introduction of additional services or service elements into intermediate care appears to be causing even more complexity and uncertainty, thereby inhibiting, rather than promoting, holistic service provision for these particular client groups. ${ }^{13}$

\section{GP out of hours services}

Recent changes to the National Out of Hours Contract (April 2004) will mean that fewer GPs will now opt to provide out of hours services, ${ }^{14}{ }^{15}$ which must impact on the future demand for alternative emergency care services.

\section{METHOD}

\section{Literature review}

To clarify the future demands on the paramedic in the primary care setting, and to identify the basic skills, training, and professional capacity which paramedics of the future will undoubtedly require, the evidence concerned with paramedic practice was explored by an indepth search of literature published between January 1995 and April 2004. Data sources accessed are shown in box 2. The search terms (box 3) used were wide and varied because of the lack of publications evidenced in this field of study.

To complement the literature search and further develop the knowledge base, a series of informal discussions with staff of the South Yorkshire Ambulance Service and Sheffield Hallam University, the Workforce Development Team (NHS Modernisation Agency), and researchers currently active in the field of emergency care were also undertaken.

\section{The nature of the evidence base}

Despite widespread acknowledgement of the value of the role of the paramedic in managing prehospital crises, this review found the body of high quality evidence with which to

\section{Box 2: Data sources searched}

- Online search facilities of Medline, Embase, Biome, CINHAL, Cochrane, NELH (Emergency Care Section), NHS/Department of Health sites, National Institute of Clinical Excellence (NICE), York Centre for Reviews and Dissemination

- Online journals: BMJ, Emergency Medicine Journal, Journal of Emergency Medicine, Journal of International Emergency Medicine, American Journal of Emergency Medicine, Prehospital Emergency Care, Annals of Emergency Medicine

- Handsearch of 2003-04 issues of the Emergency Medicine Journal

- Sheffield University Centre for Health and Related Research (ScHARR) and Medical Research Unit (MCRU) website and publications

- Warwick/Birmingham universities and Coventry NHS Trust (EmERG) websites and publications

- UK/US/Australian/Canadian ambulance services websites

- Internet search engines (Google and MSN)

- Reference lists of reviewed articles were accessed and relevant articles were also searched.

- Specific initiatives and government agencies were searched for by name. 


\section{Box 3: Search terms used}

- Paramedic

- Role of paramedic

- New role paramedic

- Paramedic education

- Paramedic training

- Pre-hospital care

- Emergency care

- Emergency care practitioner

- Emergency care technician

- Emergency care services

- Ambulance

- Ambulance service

- 999 calls

- 911 calls

- Telephone triage

validate key aspects of paramedic practice to be minimal. The evidence base is currently growing, gaining increased impetus with the establishment of the International PreHospital Network within the Cochrane Collaboration and the introduction of NHS clinical governance, clinical audit, and risk management initiatives. However, the number of randomised controlled trials at present described in the literature is diminutive, with few evaluating outcomes for paramedic interventions effectively. ${ }^{16}{ }^{17}$

Given the paucity of published evidence, this review comprises an overview of key articles and literature reviews by well recognised researchers in the field of emergency care. These articles were systematically collated and contextualised in combination with government documents and contemporary commentaries.

\section{RESULTS}

\section{Key findings from the evidence}

The available literature was collected and appraised and the findings explored working with the concept of the patient journey.

\section{The telephone call}

Across the UK the total number of 999 calls continues to rise annually, while the total number of incidents attended by ambulance crews is also rising. ${ }^{19} 19$ However, available evidence reveals that the majority of emergency calls do not require paramedic skills, ${ }^{20}$ hence the next logical step has been to focus upon the interception, assessment, and prioritisation of calls, in conjunction with the provision of more appropriate responses, which better recognise patient needs. To date, systematic reviews of research into the ability of ambulance service staff to triage patients by telephone to alternative models of care have repeatedly acknowledged weak research methodologies and hence, inconclusive findings. ${ }^{21}$ There are, however, several well validated studies in this field which focus on nurse led triage and management of non-urgent calls in a central control room, and these have concluded that telephone advice is safe, acceptable to callers, and cost effective to service providers. ${ }^{22}$

There remains very little high quality published evidence to quantify the effect of call prioritisation in terms of patient outcomes, and attempts at the analysis of existing literature have been hindered by the wide variety and type of telephone triage criteria and outcome measures utilised. It has also been suggested that the initiation of new research in this field has temporarily stalled, due to the imposition of National Response Time Standards across the UK ambulance services. These targets appear to have led the Service to focus more on the "manipulation and improvement" of performance data per se, rather than the initiation of improvements in patient handling and subsequent patient care. ${ }^{23}$ The lack of contemporary evidence to link such targets with improvements in patient survival rates has now been acknowledged however, ${ }^{24}$ and the search for more appropriate performance indicators continues.

\section{At the incident scene}

Around the globe, the validating evidence for many elements of prehospital, on-scene triage by paramedics remains in short supply, and the majority of current practice is therefore founded upon anecdotal evidence and expert opinion. ${ }^{3}$ Many researchers have described the sheer breadth of possible onscene diagnoses as being beyond the grasp of current paramedic practice, ${ }^{25}$ and have particularly highlighted the consistent problems with the recognition, diagnosis, and management of "occult" (that is, hidden) conditions, minor illnesses, and trauma. ${ }^{26}$

The development and implementation of universal clinical guidelines within the ambulance service is currently a priority, however, research in this field has been described as "pseudo scientific", exhibiting little internal validity and demonstrating huge variation in outcomes. ${ }^{27}$ Many studies have found the use of clinical guidelines to be "impractical" and have repeatedly found guidelines to be misapplied in practice settings, with a large proportion of conditions, or severity of conditions, being misdiagnosed..$^{28}$ Indeed, it has been argued by some that guidelines have proved counterproductive, causing the paramedic role to evolve as a product of what doctors have made it, rather than fostering the growth of the autonomous practitioner, capable of questioning what should reasonably be done, and where. ${ }^{29}$

A small number of high quality studies, however, have shown paramedics to be capable of the successful triage of certain patients on-scene. For example, paramedics have been shown to perform well in identification of a cohort of patients with definite cardiac infarction ${ }^{30}$ and in the redirection of non-urgent callers to alternative destinations to A\&E. ${ }^{31}$ Despite these findings, many researchers feel that the overriding unacceptability of under-triage at the incident scene (and, to a lesser extent, the undesirably high costs of over-triage), ${ }^{32}$ which is present within today's "risk managed" service means that future research efforts would be more effective if they focus instead upon identifying and eliminating the primary barriers to healthcare access which are associated with inappropriate calls to the 999 service.

\section{The paramedic practitioner}

There is a developing body of research into the piloting of the paramedic practitioner role in a wide range of settings, but this proved difficult to collect and appraise with rigour, due to its ongoing nature and lack of formal publication. Nevertheless, results from "work in progress" appear promising.

The East Anglian Ambulance Service Trust is currently evaluating the community paramedic role, and a published review of its findings to date has shown a dramatic reduction in A\&E admissions coupled with improvements in 999 response times. ${ }^{33}$ The South Yorkshire Ambulance Service has also invested in the training of community paramedics, who are employed in a trial of an intermediate care support scheme. Early indications are promising and show that over half of older patients with minor injuries are now being treated in their own homes. ${ }^{34}$ Trials of the emergency care practitioner role by both the London Ambulance Service and 
the West Country Ambulance Service have revealed patients to be receiving "more appropriate care more rapidly".

\section{Maintaining the paramedic workforce}

Job satisfaction and personal wellbeing among paramedics are undoubtedly important issues, which must come to the fore. Perceptions of new roles and ongoing changes in service delivery are varied, and discussions within Workforce Development Team focus groups revealed that not all practitioners regard such innovation as the "exciting opportunity" portrayed in the media.

National Response Times have meant that the majority of ambulance services now use continuous vehicle mobilisation between caller "hot spots", with staff no longer able to spend time between calls on the station, relaxing and socialising with colleagues. As a consequence of this new way of working, levels of stress related illness are increasing within the service, ${ }^{35}$ while concerns surrounding the lack of scope for post-trauma counselling and debriefing continue to grow. Development of the clinical supervisor's role may provide one possible support mechanism.

Increases in the incidence of postural, musculoskeletal pain from prolonged sitting in a vehicle on stand-by have also been identified as recurrent problematic areas within the field of occupational health. Although published research in this field remains minimal, the South Yorkshire Ambulance Service is among a small number of providers involved in the evaluation of a "fast track" physiotherapy service for employees with persistent back pain. ${ }^{36}$

\section{DISCUSSION AND RECOMMENDATIONS Service commissioners}

If ambulance services are to move towards becoming part of a fully integrated care delivery system with access to numerous service "portals", partnership is the key to the future, and a great deal of work is ongoing to respond to this challenge across the UK.

A large proportion of the published research has been aimed at reducing inappropriate patient transport and has targeted improvements in on-scene triage by the implementation of clinical guidelines. However, a combination of National Service Response Times and the medicolegal environment mean that, at present, such interventions have a limited potential for savings, both in terms of service deployment and relative costs. Future research effort, therefore, might prove more constructive if it focused upon raising the profile of new service options in both public and training arenas, while also further exploring the seminal inequalities in service access that still remain among vulnerable minority populations.

\section{Service providers}

The key to the future provision of prehospital care is dependent on improvements in call prioritisation and triage systems, and must lie in working in parallel with other healthcare providers. There is an underlying impetus for a single NHS call prioritisation system, based upon the concept of a centralised "response generator", intercepting 999 calls, GP out of hours calls and NHS Direct/Care Direct calls and determining an appropriate, integrated service response. If ambulance services are to continue to concentrate upon the delivery of emergency care to patients' at most immediate life threatening risk, they must develop specialist resources to target such cases, whereas callers who do not require a specialist response should be more appropriately redirected to alternative points of health service access.

In the immediate future therefore there will undoubtedly be a need for paramedic practitioners, possibly based within a primary care setting, who are capable of the accurate recognition of a wide range of both adult and paediatric conditions, carrying out rapid on-scene assessments, instituting advanced treatments in line with evidence based guidelines, and fast-tracking patients in a stable condition to the nearest, most appropriate, destination.

\section{Educationalists}

Hopefully this review has served not only to clarify the future demands which confront the emergency services in the primary care setting, but has also identified the core skills, training, and professional development which paramedics of the future will require. However, the opportunities for paramedic development vary considerably across the UK, possibly as a corollary of the lack of valid research evidence with which to support their skills in practice. Nevertheless there is little doubt that the ambulance services of the future must accommodate certain key paramedics, capable of operating as an independent practitioners and trained to a much higher level in patient assessment and decision making skills. In their turn, complementary health service staff must be prepared to both accept and respect their newfound autonomy.

National links between paramedic educational programmes remain underdeveloped, and some continue to target the development of managerial skills rather than clinical competence, thus failing to provide a sound basis for uniform career foundation. The lack of involvement of paramedics in service changes also does not bode well for the future. To reassert their rightful place in the provision of prehospital care, practitioners must be educated and encouraged to take a far broader, all encompassing view of their practice and its effect upon patient outcomes.

\section{CONCLUSION}

The UK ambulance service today remains under increasing and unremitting pressure, with both the number of incidents attended and the predicted demand for patient journeys continuing to rise. Within this setting, the service is compelled to move away from being a relatively uncomplicated patient transport system towards becoming part of a fully integrated, national healthcare service, to "do things differently", and thereby facilitate a fully coordinated and holistic approach to patient handling in the community. It becomes clear that within this framework the paramedic of the future must be able to effectively diagnose a far wider range of conditions, institute treatment according to national evidence based guidelines, reflect upon and critically evaluate the effectiveness of care, determine when and if specific interventions should be used, and provide optimum care solutions which are unique and specifically tailored to each patient.

Holistic ways of working which take a far wider approach to service provision are the long term goal therefore and, as such, must impact upon the future training of the paramedic. Paramedic training must move its focus away from the acquisition of clinical skills (no matter how wide ranging), towards fostering the ability to think and practise autonomously. In the short term, paramedics must be taught to appropriately diagnose and manage a wider range of patients, particularly those who present with occult (hidden) conditions, minor illnesses, and minor trauma. In the longer term, and more importantly however, they must learn to work together to take ownership of the basic philosophies of their practice, which are solidly grounded in methodologically valid and reliable research, and therefore truly "evidence based". 


\section{ACKNOWLEDGEMENTS}

The author would particularly like to acknowledge the help of Clinical Governance/Audit Staff, paramedic practitioners, paramedics, and Clinical Supervisors at the South Yorkshire Ambulance Service, and paramedic tutors at Sheffield Hallam University.

Competing interests: none declared

This article presents a summary of a literature review produced by Sheffield Hallam University in collaboration with the South Yorkshire Ambulance Service. Copies of the complete review are available by contacting the author at the School of Health and Wellbeing, or by e-mail from l.ball@shu.ac.uk

\section{REFERENCES}

1 Woolard M, Ellis E. Pre-hospital care five years hence. Pre-hosp Immediate Care 1999:3:102-7.

2 Audit Commission. A life in the fast lane: Value for money in emergency ambulance services. London: Audit Commission, 1998.

3 Lord B. Reconfirming the role of paramedics in pre hospital care. Australas J Emerg Care 1998:5:10-14.

4 Baskett $P$, Chamberlain D, Clarke T, et al. The future role and education of Ambulance Service Personnel (Emerging concepts). London: Joint Royal Colleges Ambulance Service Liaison Committee and Ambulance Service Authority Publication, 5 January 2000.

5 O'Cathain A, Turner J, Withers A, et al. Views of people who call 999 to request an ambulance. Pre-hosp Immediate Care 1999;3:131-5.

6 Wardrope J, Driscoll P. Turbulent times: the pace of reform may exceed capacity. Emerg Med J 2003;20:116.

7 Sakr M. Angus $\mathrm{H}$, Perrin J, et al. Care of minor injuries by emergency nurse practitioners or junior doctors: A RCT. Lancet 1999;354:1321-6.

8 Lindley-Jones M, Finlayson BJ. Triage Nurse requested Xrays-Are they worthwhile? J Accid Emerg Med 2000;17:103-7.

9 Cooke MW, Higgins J, Bridge P. Minor injury services: The present state. EmERG: University of Warwick and Birmingham, 2000.

10 Audit Commission. The coming of age: Improving care services for older people. London: Audit Commission, 1997.

11 National Bed Enquiry. Response to a consultation exercise on the National Bed Enquiry. London: DoH, 2001

12 Department of Health. Intermediate Care: Moving Forwards. National service framework for older people. London: DoH, 2002

13 Petch A. Intermediate Care: What do we know about old people's experiences?. Glasgow: University of Glasgow, Joseph Rowntree Foundation Publications, 2003.

14 Neal R. GP's reluctant to cover out of hours work [rapid response]. BMJ 2004;328:247

15 Koralage N. GPs reluctant to cover out of hours work, survey shows [news roundup]. BMJ 2004;328:247.

16 Callaham M. Quantifying the scanty science of pre-hospital emergency care. Ann Emerg Med 1997;30:785-90.
17 Kwan I, Bunn F, Roberts I, et al. The development of a register of randomized controlled trials in prehospital trauma care. Prehosp Emerg Care 2002;6:27-30

18 Wrigley H, Snooks H, Thomas E, et al. Epidemiology and demography of 999 ambulance calls: A review. Pre-hosp Immediate Care 1999;3:4-98.

19 Dale J, Crouch R, Visavadia B, et al. Provision of telephone advice from A\&E departments: A national survey. J Accid Emerg Med 1999;16:112-13.

20 Hassan TB, Barnett DB. Delphi type methodology to develop consensus on the future design of Emergency Medical Systems in the UK. Emerg Med J 2002; 19:155-9.

21 Snooks H, Foster T, Nicholl J. Results of an evaluation of the effectiveness of triage and direct transportation to minor injuries units by ambulance crews. Emerg Med J 2004;21:105-11.

22 Dale J, Higgins J, Williams S, et al. Computer assisted assessment and advice for non serious 999 ambulance service callers: the potential impact on ambulance despatch. Emerg Med J 2003;20:178-83.

23 Commission for Health Improvement. What CHI has found in Ambulance Trusts. London: CHI Publications, 2003.

24 Callaham M. Quantifying the scanty science of prehospital emergency care. Ann Emerg Med 1997;30:785-90.

25 Bissell RA, Seaman KG, Bass RR. Change the scope of practice of paramedics? An EMS Public Health Policy perspective. Prehosp Emerg Care 1999:3:140-9.

26 Silvestri S, Rothcock SG, Kennedy E, et al. Can paramedics accurately identify patients who do not require emergency department care? Prehosp Emerg Care 2002;6:387-90

27 Cone DC, Benson R, Scmidt TA, et al. Field Triage Systems: Methodologies from the literature. Prehosp Emerg Care 2004;8:28-42.

28 Snooks HA, Dale J, Hartley-Sharpe C, et al. On scene alternatives for emergency ambulance crews attending patients who do not need to travel to the accident and emergency department: a review of the Literature. Emerg Med J 2004;21:212-15.

29 Warwick J. Paramedic agrees with most comments about pre-hospital care [letter]. BMJ 2000;320:1005

30 Quinn T, Allen TF, Thompson DR, et al. Identification of patients suitable for direct admission to coronary care unit by ambulance paramedics. Pre-hosp Immediate Care 1999;3:126-30.

31 Schaefer RA, Rea TD, Porde $M$, et al. An emergency medical services program of alternate destination of patient care. Prehosp Emerg Care 2002;6:309-14.

32 Asplin BR. Under-triage, over-triage or no triage? In search of the unnecessary emergency department visit. Ann Emerg Med 2001;38:282-5.

33 Everden E, Bilby $M$, et al. Community paramedics. Available at: www.eastanglianambulance.com (accessed April 2004).

34 Mason S, Wardrope J, Perrin J. Developing a community paramedic practitioner intermediate care support scheme for older people with minor conditions. Emerg Med J 2003;20:196-8.

35 Bennett P, Williams Y, Page N, et al. Levels of Mental Health problems amongst UK emergency ambulance workers. Emerg Med J 2004;21:235-6.

36 Frisby J, Mawson S. Proposal for a fast-track physiotherapy service for SYAS. Sheffield: Sheffield Hallam University, 2002. 